\title{
Salicylic Acid-Mediated
}

\section{Diacylglycerol/Triacylglycerol Conversion Affects the Freezing Tolerance of Arabidopsis}

\author{
Le Xu (D 597582012@qq.com ) \\ Yangtze University \\ Jun Wu \\ Yangtze University \\ Yancui Zhao \\ Yangtze University \\ Huaqiong Liu \\ Yangtze University \\ Wenying Zhang \\ Yangtze University \\ Yanhao Xu \\ Yangtze University
}

\section{Research Article}

Keywords: salicylic acid, diacylglycerol, triacylglycerol, cold stress, Arabidopsis

Posted Date: December 21st, 2021

DOI: https://doi.org/10.21203/rs.3.rs-1091252/v1

License: (9) (i) This work is licensed under a Creative Commons Attribution 4.0 International License.

Read Full License 


\section{Abstract}

Diacylglycerol (DAG) is likely converted to triacylglycerol (TAG) by the enzyme diacylglycerol acyltransferase (DGAT), and this conversion is important in freezing tolerance of Arabidopsis. The phytohormone salicylic acid (SA) plays important roles in the chilling and freezing tolerance of plants. In our study, we analysed the chilling phenotype, proline and sugar accumulation, phytohormone measurement, and lipid profiling of dgat1 mutants during chilling or freezing stress. We found that dgat11 mutants exhibited higher sensitivity to long exposure to cold stress and showed lower proline and sugar accumulation under cold acclimation conditions. The freezing-sensitive phenotype of dgat1 mutants can be ameliorated by mutations of key salicylic acid (SA) signalling components SAG101, EDS1, and PAD4 through phenotyping analysis of double mutants. Dgat1 mutants accumulated more SA, ABA, JA-lle (jasmonate isoleucine) and OPDA (12- oxyphytodienoic acid) during freezing stress and after recovery. In addition, the DAG/TAG content in the SA-deficient mutant sid2 was lower than that in the wild type, while the SA-excessive accumulated mutant siz1 showed the opposite trend. In summary, SA could mediate the freezing tolerance of Arabidopsis by regulating the ratio of DAG and TAG, which influences the integrity of the membrane.

\section{Introduction}

Cold stress can be divided into chilling (temperature above $0^{\circ} \mathrm{C}$ ) and freezing stress (temperature lower than $0^{\circ} \mathrm{C}$ ), which are major environmental factors that severely restrict plant growth, development, productivity, and geographical distribution (Thomashow, 1999). Most temperate plants have evolved complex strategies, such as the accumulation of cryoprotective polypeptides and osmolytes (e.g., soluble sugars and proline), transcriptome reprogramming, and remodelling of biochemical membranes to cope with low temperatures (Thomashow 1999). Prior exposure to nonfreezing temperatures allows plants to survive freezing stress and involves the activation of several physiological processes to enhance the plant tolerance to freezing temperatures (Thomashow, 1999) and this process is called cold acclimation.

Recent work has shown that the defence hormones salicylic acid (SA), ethylene (Shi et al., 2012), and jasmonic acid (JA) modulate cold stress responses in Arabidopsis. JAs function as critical upstream signals in the ICE-CBF/DREB1 pathway and they positively regulate Arabidopsis freezing tolerance through the JASMONATE ZIM-DOMAIN (JAZ) proteins JAZ1 and JAZ4 (Hu et al. 2013). Unlike the immediate accumulation of JAs in Arabidopsis seedlings after exposure to cold stress, SA levels increased only after a week of being kept at cold temperatures (Scott et al. 2004; Shi et al., 2012; Hu et al. 2013; Kim et al. 2013). Nonetheless, cold stress induces an increase in endogenous SA, and the existing evidence suggests that there is a strong negative correlation between the growth rate under cold conditions and the levels or perception of SA (Scott et al. 2004). The wild type suffers more stagnant growth than the SA-deficient mutant NahG and eds5 (inhibited SA transport from the chloroplast) mutants under cold stress, and the partially SA-insensitive $n p r 1$ mutant (a nonexpresser of SA-inducible PR genes) displays growth intermediate between that of NahG and wild type (Scott et al. 2004, Kim et al. 2013). In addition, the SA-deficient mutants eds5 and sid2 (chloroplastic SA synthesis deficient) had 
higher survival rates during recovery after freezing stress (Kim et al. 2013; Majláth et al. 2011). In contrast, the cpr1 mutant (constitutive expresser of PR genes) that accumulated very high levels of SA at $5^{\circ} \mathrm{C}$ showed more inhibited growth than the wild type. In addition, the accumulation of SA in siz 1 and acd6 mutants confers hypersensitivity to freezing stress (Miura and Ohta, 2010).

Ice formed during freezing stress could cause substantial damage to the cell membrane. Thus, membrane integrity is a vital factor influencing the freezing tolerance of plants, and ion leakage is a reliable indicator of membrane damage (Chen and Thelen, 2013). Lipids are important components of the membrane, and the species, ratio, and remodelling of different lipids could determine the freezing tolerance of plants. Increasing evidence suggests that both plastidic and extraplastidic membrane lipid alterations affect membrane fluidity and integrity in plants exposed to cold stress. (Uemura et al., 1995; Welti et al., 2002; Li et al., 2004; Moellering et al., 2010; Chen and Thelen, 2013). For example, in Arabidopsis rosettes, freezing induces significant decreases in phosphatidylcholine (PC) and phosphatidylethanolamine (PE), as well as plastidic monogalactosyldiacylglycerol (MGDG) and phosphatidylglycerol (PG), while phosphatidic acid (PA) and lysophospholipids increase dramatically (Welti et al., 2002). MGDGs have a small head group, and PA lacks a head group; they are inclined to form the inverted hexagonal II (HII)-type phase, and these nonlamellar lipid structures can drive the disruption of cell membrane integrity (Verleij et al., 1982; Moellering et al., 2010; Steponkus, 1984; Uemura et al., 1995; Thomashow, 1999; Welti et al., 2002).

In Arabidopsis, disruption of SFR2 (sensitive to freezing tolerance), which encodes a galactolipid:galactolipid galactosyltransferase (GGGT) that converts MGDG to oligogalactolipids (such as diacylglycerol, DAG), results in severe injury during freezing stress (Thorlby et al., 2004; Moellering et al., 2010; Fourrier et al., 2008). Furthermore, DAG produced by GGGT can be subsequently converted to triacylglycerol (TAG) by acyl-coenzyme a diacylglycerol acyltransferase (DGAT), likely resulting in the removal of excess adverse membrane lipids after freezing (Moellering et al., 2010; Moellering and Benning, 2011). Phosphorylation of DAG by DAG kinases (DGKs) can produce PA (Wang, 2004), and DGKs transcripts are induced rapidly in response to chilling stress (Go'mez-Merino et al., 2004; Arisz et al., 2013).

The conversion of DAG to TAG by DGAT1 is critical for plant freezing tolerance by balancing TAG and PA production in Arabidopsis. The dgat1 mutants are more sensitive to freezing stress than the wild type. The blockage of DAG to TAG conversion in the dgat1 mutant activates the DAG to PA conversion pathway through the function of DGKs. During freezing stress, the accumulation of PA in dgat1 plants stimulated NADPH oxidase activity and enhanced RbohD-dependent hydrogen peroxide production compared with the wild type. Consistent with this observation, $d g k 2, d g k 3$, and $d g k 5$ knockout mutants showed attenuated PA production and improved tolerance in response to freezing temperatures (Tan et al., 2018). The sag101, eds1, and pad4 mutants had much lower SA and DAG contents than the wild type, and exogenous application of SA and DAG compromised the freezing tolerance of the mutants.

Furthermore, SA suppressed the cold-induced expression of DGATs and DGKs in wild-type leaves. These 
findings indicate that SAG101, EDS1, and PAD4 are involved in the freezing response in Arabidopsis, at least in part, by modulating the homeostasis of SA and DAG (Chen et al., 2015).

However, it is still unknown how the enzymes downstream of DAG contribute to the balance of TAG and PA production under freezing conditions, and the precise cellular functions of Arabidopsis DGKs remain elusive. Based on previous reports and references, the purpose of this work was to elucidate how the phytohormone SA regulates DAG-TAG conversion and the cold tolerance of Arabidopsis.

\section{Materials And Methods}

The Arabidopsis ethyl methanesulfonate-generated mutant dgat1-1 (CS3861, a gift from Shi Lei of oil crops research institute, Chinese Academy of Agricultural Science) and T-DNA insertional mutant dgat12 (SALK_039456) were the same as the seeds used by Tan et al (2018).

Seeds of Arabidopsis wild type (ecotype Columbia-0) and mutants were surface sterilized with $20 \%$ bleach $(\mathrm{v} / \mathrm{v})$ for $15 \mathrm{~min}$ and then washed three times with sterilized water followed by sowing on MS medium supplemented with $1 \%$ sucrose. Seeds were dark treated at $4{ }^{\circ} \mathrm{C}$ for $3 \mathrm{~d}$ and subsequently transferred to plant growth room under a 16 -h light $\left(23^{\circ} \mathrm{C}\right) / 8$-h dark $\left(21^{\circ} \mathrm{C}\right)$ cycle. After germination for 8 days, the seedlings were transplanted to soil in the plant growth room until treatment. Chilling and freezing treatments were carried out as described previously (Chen et al. 2015) with minor modifications. For cold acclimation and chilling treatment, 4-week-old plants were transferred from the growth room to a cold growth chamber $\left(4^{\circ} \mathrm{C}\right)$ for $3 \mathrm{~d}$ of acclimation or several weeks under a normal light/dark cycle, respectively. For the freezing treatment, all 4-week-old NA (non-acclimated) or CA (cold-acclimated) soilgrown plants or 11-d-old seedlings grown on MS medium plates were transferred to a growth chamber (Blue Pard LRH- LRH-500CA) with temperatures reduced steadily from $4^{\circ} \mathrm{C}$ to $-2{ }^{\circ} \mathrm{C}\left({ }^{\circ} \mathrm{C}\right.$ per hour). The temperature remained at $-2^{\circ} \mathrm{C}$ for $2 \mathrm{~h}$ and continued to decrease until reaching the final temperatures. After staying at the final temperatures for 1 hour, the plants were thawed overnight at $4{ }^{\circ} \mathrm{C}$. Plants were photographed after a 5 -d recovery under normal growth conditions.

\section{Lipid Profiling}

Leaf samples in chloroform/methanol/water $(2 / 1 / 1, \mathrm{v} / \mathrm{v} / \mathrm{v})$ solution were vortexed for $1 \mathrm{~min}$, and centrifuged at $3000 \mathrm{rpm}$ for $10 \mathrm{~min}$. Organic phage was collected and transferred to a new tube and lyophilized using nitrogen. Dried metabolites were reconstituted in $400 \mathrm{ul}$ isopropanol/methanol $(1 / 1, v / v)$ solution, vortexed, centrifuged at $12000 \mathrm{rpm}$ for $10 \mathrm{~min}$ at $4{ }^{\circ} \mathrm{C}$ and supernatant was analyzed using LC-MS. A Kinetex C18 column $(100 \times 2.1 \mathrm{~mm}, 1.9 \mu \mathrm{m})$ and the following gradient: $0-2$ min 30\% mobile phase $B ; 2-20$ min $100 \%$ B; $20-40$ min $100 \%$ B; $40-40.01$ min 30\% B; 40.01-45 min $30 \% \mathrm{~B}$, was applied for the experiment. Mobile phase $A$ was acetonitrile/ water $(3: 2, \mathrm{v} / \mathrm{v}), 10 \mathrm{mM}$ ammonium formate. Mobile phase $B$ was acetonitrile/ isopropanol $(1: 9, \mathrm{v} / \mathrm{v}), 10 \mathrm{mM}$ ammonium formate and $0.1 \%$ formic acid. The flow rate was $0.4 \mathrm{ml} / \mathrm{min}$, the column was at $45^{\circ} \mathrm{C}$. Parameters used for mass spectrometry were described as follows: positive ion mode, Heater Temp $300^{\circ} \mathrm{C}$, Sheath Gas Flow rate, 45arb, Aux Gas Flow Rate, 15 arb, Sweep Gas Flow Rate, 1arb, spray voltage, 3.0KV, Capillary Temp, 350 
${ }^{\circ} \mathrm{C}$, S-Lens RF Level, 30\%. Scan ranges:200-1500; negative ion mode, Heater Temp300 ${ }^{\circ} \mathrm{C}$, Sheath Gas Flow rate, 45arb, Aux Gas Flow Rate, 15arb, Sweep Gas Flow Rate, 1arb, spray voltage, 2.5KV, Capillary Temp, $350^{\circ} \mathrm{C}$, SLens RF Level, $60 \%$. Scan ranges: $200-1500$. Lipidomics assays was performed in Biocluster Inc.

\section{Extraction and Measurement of Phytohormone Levels}

The levels of ABA, SA, JA, and OPDA were determined by Zoonbio Biotechnology Co., Ltd., and the methods were modified from those described by Pan et al (2002). Approximately $0.5 \mathrm{~g}$ leaves were ground in a precooled mortar that contained $\mathrm{mL}$ extraction buffer comprised of isopropanol/hydrochloric acid. The extract was shaken at $4{ }^{\circ} \mathrm{C}$ for $30 \mathrm{~min}$. Then, $10 \mathrm{ml}$ dichloromethane was added, and the sample was shaken at $4{ }^{\circ} \mathrm{C}$ for $30 \mathrm{~min}$ and centrifuged at $13,000 \mathrm{rpm}$ for $5 \mathrm{~min}$ at the same temperature. We then extracted the lower, organic phase. The organic phase was dried under $\mathrm{N}_{2}$ and dissolved in $150 \mu \mathrm{L}$ methanol (0.1\% methane acid) and filtered with a $0.22-\mu \mathrm{m}$ filter membrane. The purified product was then subjected to high-performance liquid chromatography-tandem mass spectrometry (HPLC-MS/MS) analysis. HPLC analysis was performed using a ZORBAX SB-C18 (Agilent Technologies) column ( $2.1 \mathrm{~mm} \times 150 \mathrm{~mm} ; 3.5 \mathrm{~mm})$. Mobile phase A solvents consisted of methanol/0.1\% methanoic acid, and mobile phase B solvents consisted of ultrapure water/0. $1 \%$ methanoic acid. The injection volume was $2 \mu \mathrm{L}$. The MS conditions were as follows: the spray voltage was $4500 \mathrm{~V}$; the pressure of the air curtain, nebulizer, and aux gas was 15,65 , and 70 psi, respectively; and the atomizing temperature was $400^{\circ} \mathrm{C}$.

\section{Proline and Soluble Sugar Contents Measurement.}

Soluble sugar measurements were carried out according to a previous report (Li et al. 2004). The rosette leaves were harvested, weighed, ground into fine powder in liquid nitrogen and then incubated in $75 \%$ ethanol overnight with gentle shaking. After centrifugation at $20,000 \mathrm{~g}, 20 \mu \mathrm{L}$ of each extract was incubated with $1000 \mu \mathrm{L}$ of anthrone reagents $\left[0.15 \%(\mathrm{w} / \mathrm{v})\right.$ anthrone, $72 \%(\mathrm{v} / \mathrm{v}) \mathrm{H}_{2} \mathrm{SO}_{4}$, and $28 \%(\mathrm{v} / \mathrm{v})$ water] at $100^{\circ} \mathrm{C}$ for I h. The soluble sugar value was measured at $630 \mathrm{~nm}$.

Proline measurement was carried out according to a previously described method (Bates et al. 1973). The rosette leaves were weighed and subjected to extraction using $3 \%$ sulfosalicylic acid. After filtration, $2 \mathrm{~mL}$ of each filtrate was incubated with $2 \mathrm{~mL}$ of glacial acetic acid and $2 \mathrm{~mL}$ of acid ninhydrin reagent [2.5\% (w/v) ninhydrin, $60 \%(\mathrm{v} / \mathrm{v})$ glacial acetic acid, and 40\% (v/v) $6 \mathrm{M}$ phosphoric acid] at $100{ }^{\circ} \mathrm{C}$ for $1 \mathrm{~h}$. The reaction was terminated in an ice bath. Toluene $(4 \mathrm{~mL})$ was added to the extraction followed by vigorous shaking for $20 \mathrm{~s}$. After incubation at $23^{\circ} \mathrm{C}$ for $24 \mathrm{~h}$, the proline values were measured at $520 \mathrm{~nm}$.

\section{Results}

\section{Dgat1-1 mutant exhibit enhanced chilling sensitivity}


When 3-week-old plants were exposed to $4{ }^{\circ} \mathrm{C}$ for several weeks, the rosettes of dgat $1-1$ mutants became yellowish when it was exposed to chilling stress for 4 weeks compared with the wild-type rosettes under the same conditions (Figure 1). This result is consistent with previous reports of Tan et al (2018) and dgat1 mutants exhibit enhanced sensitivity to freezing stress (data not shown).

\section{Contents of Proline and Sugars were Decreased in DGAT1 Mutants under Cold Conditions}

In Arabidopsis, osmolytes such as proline and soluble sugars accumulate in response to cold stress, which may help reduce cellular damage from dehydration during freezing (Xin and Browse 1998). In this study, the contents of proline, glucose and fructose in wild-type, dgat1-1, and dgat1-2 mutants after 3 days of cold accumulation and normal conditions were measured (Figure 2). The proline content in the CA group was generally higher than that in normal (non-cold acclimation, NA) plants. The contents of glucose and proline in both dgat1 mutants were lower than those in the wild type under normal conditions and under cold acclimation. The content of fructose in the dgat1-2 mutant was lower than that in the wild type under normal growth conditions, but there was no significant difference after cold acclimation. Lower accumulation of proline and carbohydrates during cold acclimation may contribute to the sensitive phenotype of the dgat1 mutants.

\section{Phenotypic analysis of DGAT1/SAG101 double mutants.}

Previous studies found that dgat1 mutants are sensitive to freezing and chilling stress, while SA-deficient mutants sag101-3 (senescence-associated gene101), eds1-22 (enhanced disease susceptibility 1), and pad4-1 (phytoalexin deficient4) exhibit enhanced tolerance to freezing stress (Chen et al., 2015; Tan et al., 2018). We obtained similar results (data not shown). In this study, dgat1-1 and sag101-3, eds 1-22 and pad4-1 double mutants were constructed, and homozygous double mutants were used for freezing tolerance analysis. No significant differences were found between the wild type, single mutant, and double mutant under normal growth conditions. After freezing treatment and 7 days after recovery, the dgat1-1 single mutant was more sensitive than the wild type, the freezing tolerance of the double mutant was between those of the two single mutants, and the dry weight and survival ratio also support this conclusion (Figure 3). This indicates that SAG101-3, EDS1-22 and PAD4-1 mutations can recover the freezing tolerance of dgat1 mutants at least partially. We can speculate that the DAG to TAG conversion in these mutants was an important factor affecting freezing resistance and are possibly mediated by SA.

\section{Freezing-Induced Phytohormone Changes in dgat1 Mutants}

The phytohormones SA, jasmonates (jasmonic acid, JA-lle, OPDA), and ABA play important roles in regulating plant responses to chilling and freezing temperatures in Arabidopsis (Scott et al. 2004; Miura and Ohta, 2010; Shi et al., 2012; Hu et al. 2013; Kim et al. 2013; Shinkawa et al., 2013). We measured the endogenous levels of free SA and jasmonates in the rosettes of wild-type, dgat1-1, and dgat1-2 plants under normal growth conditions (NA) upon freezing treatment and recovery. As shown in Figure 4, the levels of free SA increased slightly after freezing exposure, and the dgat1 mutants showed enhanced accumulation of SA under NA and freezing conditions, whereas no significant changes in ABA, JA, JA-lle, 
or OPDA were observed between NA and the freezing plants. After recovery, the contents of ABA, SA, and jasmonates increased remarkably, and the levels of SA, JA, and OPDA were higher in the dgat1 mutants than in the wild type, while the contents of ABA and JA-lle were comparable between the wild type and mutants.

\section{Changes in Lipid Species in Wild-Type and DGAT1 Mutants Following Freezing Treatment}

To further confirm that SA might affect freezing tolerance by regulating DAG and TAG in the DGAT1 mutants, we measured DAG and TAG content in SA-deficient sid2 and SA-overexpressing siz1 mutants (Miura and Ohta, 2010) under normal growth conditions $\left(22^{\circ} \mathrm{C}\right)$ and freezing treatment $\left(-8{ }^{\circ} \mathrm{C}\right)$. At $22^{\circ} \mathrm{C}$, we observed little difference in either DAG or TAG levels between the wild type and mutants (Figure 5, detail species of DAG and TAG are not shown). In contrast, after freezing treatment, the DAG and TAG levels were dramatically elevated in all plants compared to the untreated control (Figure 5). Interestingly, after freezing treatment, the accumulation of DAG was lower in the sid2 mutant than in the wild type, and siz1 showed a more remarkable increase in DAG; accordingly, the trend of TAG accumulation was in contrast to DAG in the sid2 and siz1 mutants compared to the wild type. We can speculate that higher SA content could inhabit the DAG to TAG conversion.

\section{Discussion}

Previous studies indicate that the cold-inducible transcripts of $D G K 2, D G K 3$, and $D G K 5$ were significantly more upregulated and thus the PA content was higher in dgat 1 mutants than in the wild type during cold stress. Moreover, dgat1 mutants showed stimulated NADPH oxidase activity and enhanced RbohDdependent hydrogen peroxide production compared with the wild type. These findings demonstrate that the conversion of DAG to TAG by DGAT1 is critical for plant freezing tolerance, and plants tend to choose the DAG to PA pathway when the DAG to TAG conversion pathway is blocked (Tan et al., 2018).

Phytohormone SA could regulate the level of phospholipids. For example, previous research shows that SA induces an increase in phosphoinositides $(\mathrm{PI})$ with no increase in PA (the phosphorylated derivative of DAG, a phospholipase $C$ product), which likely reflects an in vivo inhibition of PI-PLC by SA. Ruelland et al. proposed a model in which SA inhibits PI-PLC activity and alters the levels of PI-PLC products and substrates, thereby divergently regulating gene expression (2014). In addition, previous studies have shown that SA activates PLD in Arabidopsis cell suspension cultures (Krinke et al., 2009).

SA has a contradictory role in cold stress tolerance. High-dose SA treatment causes stress, while exogenous SA treatment can protect plants against chilling stress at optimal doses. SA and other phenol derivatives are known to improve the cold tolerance of plants such as maize, banana, cucumber, rice, and pomegranate fruit (Janda et al. 1997, 1999; Kang et al. 2007; Kang and Saltveit 2002; Sayyari et al. 2009). Other related compounds, such as BA, aspirin or coumaric acid, may also have a protective role in young maize plants against cold stress (Janda et al. 1998, Janda, Szalai et al. 2000, Horváth et al. 2002). Preliminary SA treatment could increase antioxidant enzymes, reduce lipid peroxidation through the 
inhibition of lipoxygenase activity and decrease the $\mathrm{H}_{2} \mathrm{O}_{2}$ content, leading to the maintenance of cellular membrane integrity under cold stress conditions (Janda et al. 1997, 1999; Lapenna et al. 2009; Wang et al. 2006; Sasheva et al. 2010). In addition, exogenous SA caused an increase in ice nucleation activity under cold and control conditions, similar to cold acclimation in winter wheat leaves (Taşgín et al. 2003). The SA analogue 4-hydroxybenzoic acid ameliorated the freezing tolerance of the spring wheat strain Chinese Spring that is due to reinforcement of the cell wall (Horváth et al. 2007).

SA treatment may alter endogenous SA metabolism, either by inducing de novo synthesis (Szalai et al. 2011) or by decreasing and disturbing it (Rakhmankulova, et al. 2010). DGAT1 mutants showed significantly lower levels of DAG and PA and higher levels of TAG than the wild type. The application of SA suppressed the cold-induced expression of DGATs and DGKs in wild-type leaves (Chen et al. 2015). It is conceivable that DAG may act as a common substrate for PA and TAG production upon cold exposure, the homeostasis of which may determine the plant tolerance to such stresses. However, the molecular mechanism of how SA regulates lipid remodelling and responses to chilling and freezing remains to be investigated.

\section{Declarations}

Author Contributions: LX, WZ, YX conceived and designed this paper. JW, YZ, and HL conduct the experiments. $Y X, L X$ revised the manuscript.

Funding: The work was supported by the funding from the National Natural Science Foundation of China (31901438).

Conflicts of Interest: The authors declare that no conflict of interest.

\section{References}

1. Arisz SA, Van Wijk R, Roels W, et al (2013) Rapid phosphatidic acid accumulation in response to low temperature stress in Arabidopsis is generated through diacylglycerol kinase. Frontiers in Plant Science 4:1. https://doi.org/10.3389/fpls.2013.00001

2. Bates LS, Waldren RP , Teare ID (1973) Rapid determination of free proline for water-stress studies. Plant and Soil 39(1): 205-207. https://doi.org/10.1007/BF00018060

3. Chen M, Thelen JJ (2013) Acyl-lipid desaturase2 is required for chilling and freezing tolerance in Arabidopsis. Plant Cell 25(4): 1430-1444. https://doi.org/10.1105/tpc.113.111179

4. Chen QF, Xu L, Tan WJ, et al (2015) Disruption of the Arabidopsis Defense Regulator Genes SAG101, EDS1, and PAD4 Confers Enhanced Freezing Tolerance. Molecular Plant (10): 1536-1549. https://doi.org/10.1016/j.molp.2015.06.009

5. Fourrier N, Bédard J, Lopez-Juez E, et al (2010) A role for sensitive to freezing 2 in protecting chloroplasts against freeze-induced damage in Arabidopsis. Plant Journal 55(5): 734-745. https://doi.org/10.1111/j.1365-313X.2008.03549.x 
6. Gomez-Merino FC, Brearley CA, Ornatowska M, et al (2004) AtDGK2, a novel diacylglycerol kinase from Arabidopsis thaliana, phosphorylates 1-stearoyl-2-arachidonoyl-sn-glycerol and 1, 2-dioleoyl-snglycerol and exhibits cold-inducible gene expression. Journal of Biological Chemistry 279. https://doi.org/10.1074/jbc.M312187200

7. Horváth E, Janda T, Szalai G, et al (2002) In vitro salicylic acid inhibition of catalase activity in maize: differences between the isozymes and a possible role in the induction of chilling tolerance. Plant Science 163(6): 1129-1135. https://doi.org/10.1016/S0168-9452(02)00324-2

8. Horváth E, Pál M, Szalai G, Páldi E, et al (2007) Exogenous 4-hydroxybenzoic acid and salicylic acid modulate the effect of short-term drought and freezing stress on wheat plants. Biologia Plantarum 51(3): 480-487. https://doi.org/10.1007/s10535-007-0101-1

9. Hu Y, Jiang L, et al (2013) Jasmonate Regulates the INDUCER OF CBF EXPRESSION-C-REPEAT BINDING FACTOR/DRE BINDING FACTOR1 Cascade and Freezing Tolerance in Arabidopsis. Plant Cell 25(8): 2907-2924. https://doi.org/10.2307/23598259

10. Janda T, Szalai G, Tari I, Páldi E (1997) Exogenous salicylic acid has an effect on chilling symptoms in maize (Zea mays L.) plants. In: Crop development for cool and wet Europian climate, P, Sowinski B, Zagdanska A, Aniol, and P, Klaus eds, ECSP-EEC-EAEC, Brussels, Belgium pp: 179-187.

11. Janda T, Szalai G, Antunovics Z, Ducruet JM, E Páldi (1998) Effects of Salicylic Acid and Related Compounds on Photosynthetic Parameters in Young Maize (Zea Mays L.) Plants. In Photosynthesis: Mechanisms and effects (pp: 3869-3872). Springer, Dordrecht. https://doi.org/10.1007/978-94-0113953-3_902

12. Janda T, Szalai G, Antunovics Z, Horváth E, Paldi E (2000) Effect of benzoic acid and aspirin on chilling tolerance and photosynthesis in young maize plants. Maydica 45(1): 29-33.

13. Janda T, G Szalai, IPJ (1999) Hydroponic treatment with salicylic acid decreases the effects of chilling injury in maize (Zea mays $L$ ) plants. Planta 208(2): 175-180. https://doi.org/10.1007/s004250050547

14. Kang GZ, Wang ZX, Xia KF, Sun GC (2007) Protection of ultrastructure in chilling-stressed banana leaves by salicylic acid. Journal of Zhejiang University Science B 8(4): 277-282. https://doi.org/10.1631/jzus.2007.B0277

15. Kang HM, Saltveit ME (2002) Chilling tolerance of maize, cucumber and rice seedling leaves and roots are differentially affected by salicylic acid. Physiologia Plantarum 115(4): 571-576. https://doi.org/10.1034/j.1399-3054.2002.1150411.x

16. Kim YS, Park S, Gilmour SJ, Thomashow MF (2013) Roles of CAMTA transcription factors and salicylic acid in configuring the low-temperature transcriptome and freezing tolerance of Arabidopsis. The Plant Journal 75(3): 364-376. https://doi.org/10.1111/tpj.12205

17. Krinke O, Flemr M, Vergnolle C, Collin S, Eric Rulleand (2009) Phospholipase D activation is an early component of the salicylic acid signaling pathway in Arabidopsis cell suspensions. Plant Physiol 150: 424-436. https://doi.org/10.1104/pp.108.133595 
18. Lapenna D, Ciofani G, Pierdomenico SD, Neri M, Cuccurullo C, Giamberardino MA F Cu cc urullo (2009) Inhibitory activity of salicylic acid on lipoxygenase-dependent lipid peroxidation. Biochimica et Biophysica Acta (BBA)-General Subjects 1790(1): 25-

30.https://doi.org/10.1016/j.bbagen.2008.09.007

19. Li W, Li M, Zhang W, Welti R, Wang X (2004) The plasma membrane-bound phospholipase D $\delta$ enhances freezing tolerance in Arabidopsis thaliana. Nature biotechnology 22(4): 427-433. https://doi.org/10.1038/nbt949

20. Majláth I, Szalai G, Janda T (2011) Exploration of cold signalling related to ascorbate and salicylic acid in Arabidopsis thaliana. Acta Biologica Szegediensis, 55(1), 117-118.

21. Miura K, Ohta M (2010) SIZ1, a small ubiquitin-related modifier ligase, controls cold signaling through regulation of salicylic acid accumulation. Journal of plant physiology 167(7): 555-560. https://doi.org/10.1016/j.jplph.2009.11.003 Ava

22. Moellering ER, Benning CJ (2011) Galactoglycerolipid metabolism under stress: a time for remodeling. Trends in plant science 16(2): 98-107. https://doi.org/10.1016/j.tplants.2010.11.004

23. Moellering ER, Muthan B, Benning CJ (2010) Freezing Tolerance in Plants Requires Lipid Remodeling at the Outer Chloroplast Membrane. Science 330(6001): 226-228. https://doi.org/10.1126/science.1191803

24. Pan QM, Han XG, Bai YF, Yang JC (2002) Advances in Physiology and Ecology Studies on Stored Non-Structure Carbohydrates in Plants. Chinese Bulletin of Botany 2002(01):30-38

25. Rakhmankulova ZF, Fedyaev VV, Rakhmatulina SR, Ivanov CP, Gilvanova IR (2010) The Effect of Wheat Seed Presowing Treatment with Salicylic Acid on Its Endogenous Content, Activities of Respiratory Pathways, and Plant Antioxidant Status. Russian Journal of Plant Physiology 57(6): 778783. https://doi.org/10.1134/S1021443710060051

26. Ruelland E, Pokotylo I, Djafi N, Cantrel C, Repellin A, Zachowski A (2014) Salicylic acid modulates levels of phosphoinositide dependent-phospholipase c substrates and products to remodel the Arabidopsis suspension cell transcriptome. Frontiers in plant science 5:208. https://doi.org/10.3389/fpls.2014.00608

27. Sasheva P, Szalai G, Janda T, Popova L (2010) Study of the behaviour of antioxidant enzymes in the response to hardening and freezing stress in two wheat (Triticum aestivum L.) varieties. Comptes rendus de l'Académie bulgare des sciences: sciences mathématiques et naturelles 61(2): 161-172. https://doi.org/10.1002/cplx.20289

28. Sayyari M, Babalar M, Kalantari S, Serrano M, Valero D (2009) Effect of salicylic acid treatment on reducing chilling injury in stored pomegranates. Postharvest biology and technology 53(3): 152-154. https://doi.org/10.1016/j.postharvbio.2009.03.005

29. Scott IM, Clarke SM, Mur WLAJ (2004) Salicylate Accumulation Inhibits Growth at Chilling Temperature in Arabidopsis. Plant Physiology 135(2): 1040-1049. https://doi.org/10.1104/pp.104.041293 
30. Shi Y, Tian S, Hou L, Huang X, Zhang X, Yang S (2012) Ethylene Signaling Negatively Regulates Freezing Tolerance by Repressing Expression of CBF and Type-A ARR Genes in Arabidopsis. The Plant Cell 24(6): 2578-2595. https://doi.org/10.1105/tpc.112.098640

31. Shinkawa R, Morishita A, Amikura K, Machida R, Murakawa H, Kuchitsu K, Ishikawa M (2013) Abscisic acid induced freezing tolerance in chilling-sensitive suspension cultures and seedlings of rice. BMC Research Notes 6(1): 1-14. https://doi.org/10.1186/1756-0500-6-351

32. Steponkus PL (1984) Role of the plasma membrane in freezing injury and cold acclimation. Annu.rev.plant Physiol 35(1): 543-584. https://doi.org/10.1146/annurev.pp.35.060184.002551

33. Szalai G, Horgosi S, Soós V, Majláth I, alázs EB, Janda T (2011) Salicylic acid treatment of pea seeds induces its de novo synthesis. Journal of plant physiology 168(3): 213-219. https://doi.org/10.1016/j.jplph.2010.07.029

34. Tan WJ, Yang YC, Zhou Y, Huang LP, Xu L, Chen QF, Yu LJ, Xiao S (2018) DIACYLGLYCEROL ACYLTRANSFERASE and DIACYLGLYCEROL KINASE Modulate Triacylglycerol and Phosphatidic Acid Production in the Plant Response to Freezing Stress. Plant physiology 177(3): 1303-1318. https://doi.org/10.1104/pp.18.00402

35. Taşgín E, Atící Ö, Regulation B (2003) Effects of salicylic acid and cold on freezing tolerance in winter wheat leaves. Plant physiology 177(3): 1303-1318. https://doi.org/10.1104/pp.18.00402

36. Thomashow, Michael F (1999) Plant cold acclimation: freezing tolerance genes and regulatory mechanisms. Annual review of plant biology 50(1): 571-599. https://doi.org/10.1146/annurev.arplant.50.1.571

37. Thorlby, G (2004) The sensitive to freezing 2 gene, required for freezing tolerance in Arabidopsis thaliana, encodes a beta-glucosidase. Plant Cell 16(8): 2192-2203. https://doi.org/10.1105/tpc.104.024018

38. Uemura M, Joseph RA, and Steponkus PL (1995) Cold acclimation of Arabidopsis thaliana (effect on plasma membrane lipid composition and freeze-induced lesions). Plant physiology 109(1): 15-30. https://doi.org/10.1104/pp.109.1.15

39. Verkleij AJ, Maagd RD, Leunissen-Bijvelt J, Kruijff BD (1982) Divalent cations and chlorpromazine can induce non-bilayer structures in phosphatidic acid-containing model membranes. BBA Biomembranes 684(2): 255-262. https://doi.org/10.1016/0005-2736(82)90014-1

40. Wang D, Amornsiripanitch N, Dong X (2006) A genomic approach to identify regulatory nodes in the transcriptional network of systemic acquired resistance in plants. PLoS pathogens 2(11): e123. https://doi.org/10.1371/journal.ppat.0020123

41. Wang, X. (2004). Lipid signaling. Current Opinion Plant Biology. 7:329-336.

42. Welti R, Li W, Li M, Sang Y, Biesiada H, Zhou HE, Rajashekar CB, Williams TD, Wang X (2002) Profifiling membrane lipids in plant stress responses. Role of phospholipase $D$ alpha in freezing induced lipid changes in Arabidopsis. Journal of Biological Chemistry 277(35): 31994-32002. https://doi.org/10.1074/jbc.M205375200 
43. Xin Z, Browse J (1998) eskimo1 mutants of Arabidopsis are constitutively freezing-tolerant. Proceedings of the National Academy of Sciences 95(13): 7799-7804. https://doi.org/10.1073/pnas.95.13.7799

\section{Figures}

\section{Figure 1}

\section{DGAT1 Knockout Mutants Show Increased Sensitivity to Chilling Stress.}

Images of three-week-old WT, dgat1-1, and dgat1-2 plants grown at $22^{\circ} \mathrm{C}$ before (CK, top panel) and after cold treatment $\left(4^{\circ} \mathrm{C}\right.$, bottom panels) for 1 to 4 weeks.

\section{Figure 2}

\section{Accumulation of Osmolytes in Wild Type and dgat1 mutants.}

Contents of proline and soluble sugars in WT, dgat1-1, and dgat1-2 plants under NA and CA conditions. WT, dgat1-1, and dgat1-2 plants were untreated (NA) or cold-treated for 3 days and rosettes were collected for metabolite extraction. Ribitol was added as an internal quantitative standard for GC-MS analysis. The experiments were repeated (biological replicates) four times, with similar results, and the representative data from one replicate are shown. Data are means \pm SD calculated from three technical replicates. Asterisks indicate significant differences from wild type $\left({ }^{\star} P<0.05 ;{ }^{*} \mathrm{P}<0.01\right.$ by Student's $t$-test).

\section{Figure 3}

\section{Phenotype of DGAT1/SAG101 (EDS1, and PAD4) double mutants}

Images of three-week-old WT, dgat1-1/sag101, dgat1-1/eds 1 and dgat1-1/pad4 plants grown at $22^{\circ} \mathrm{C}$ before (CK, top panel) and after freezing treatment $\left(4{ }^{\circ} \mathrm{C}\right.$, bottom panels) for 1 to 4 weeks.

\section{Figure 4}

Levels of Free SA, ABA and Jasmonates in the Rosettes of Wild-Type (WT), and dgat1 Plants. 
Free SA and SA glucosides were extracted from rosettes of 4-week-old WT, dgat1-1, and dgat1-2 plants harvested after NA, freezing treatment $\left(-8^{\circ} \mathrm{C}\right)$ for $1 \mathrm{~h}$ (Freezing) and recovery for $12 \mathrm{~h}$ (Recovery), and the extracts were analyzed by LC-MS/MS. The experiments were repeated (biological replicates) three times, with similar results, and the representative data from one replicate are shown. Data are means $\pm S D$ calculated from three technical replicates. Asterisks with "a" indicate significant differences from that of NA wild type and with "b" indicate significant differences from wild type $\left({ }^{\star} \mathrm{P}<0.05 ; \star \star \mathrm{P}<0.01\right.$ by Student's $t$-test).

\section{Figure 5}

DAG and TAG Profiles of 4-Week-Old Wild-Type (WT), dgat1-1, and dgat1-2 Rosettes Before $\left(22^{\circ} \mathrm{C}\right)$ and After CA Followed by Freezing Treatment $\left(-8^{\circ} \mathrm{C}\right)$.

Total amounts (signal/mg dry weight) of diacylglycerol (DAG; left column) and tri-

acylglycerol (TAG; right column) in WT, dgat1-1, and dgat1-2 rosettes. Data are means \pm SD calculated from four technical replicates. Asterisks indicate significant differences from wild type $\left({ }^{\star} P<0.05 ; \star \star P<\right.$ 0.01 by Student's $t$-test). 